\title{
Powder, Solvent and Matrix for Implantation Matrix Dosage Form
}

National Cancer Institute

\section{Source}

National Cancer Institute. Powder, Solvent and Matrix for Implantation Matrix Dosage

Form. NCI Thesaurus. Code C149858.

Sterile powder, sterile solvent and sterile matrix intended for the preparation of an implantation matrix, for example by dissolving the powder in the solvent and using the resulting solution to impregnate the matrix. 